\title{
A case of metastatic urachal adenocarcinoma treated with several different chemotherapeutic regimens
}

\author{
Christine Elser, MD; Joan Sweet, MD, FRCP; ${ }^{+}$Sendhil K. Cheran, MD, ${ }_{+}^{\ddagger}$ Masoom A. Haider, MD, FRCPC; ${ }^{\ddagger}$ Mi- \\ chael Jewett, MD, FRCSC; ; Srikala S. Sridhar, MD, MSc, FRCPC*
}

\begin{abstract}
*Medical Oncologist, Assistant Professor, Department of Medical Oncology and Hematology, Princess Margaret Hospital, Toronto, ON; †Pathologist, Department of Pathology, University Health Network, Toronto, ON; : Radiologist, Department of Radiology, University Health Network, Toronto, ON; 士Urologist, Department of Urology, Princess Margaret Hospital, Toronto, ON
\end{abstract}

Cite as: Can Urol Assoc J 2012;6(1):e27-31. htrp://dx.doi.org/10.5489/cuaj.11109

\section{Abstract}

Urachal cancer is a rare form of bladder cancer that arises from the urachus, a vestigial musculofibrous band that extends from the dome of the bladder to the umbilicus. Urachal cancer often presents at an advanced stage and has a dismal prognosis. We present a case of a young woman with urachal cancer that recurred rapidly post-partial cystectomy. The patient was treated with up to 6 different chemotherapy regimens, including an oral antiangiogenic drug as part of a clinical trial. Our case illustrates the aggressive nature of this disease, the difficulty in accessing drugs for the treatment of rare cancers and the lack of late stage clinical trials to help guide therapeutic decisions.

\section{Case report}

A 35-year-old woman, who was previously well, presented with an 8-month history of abdominal pain, urinary tract infections and a 4-month history of gross hematuria and bloody discharge from the umbilicus. She underwent a computed tomography (CT) scan which showed a $6.2 \times 6.0 \times 4.0 \mathrm{~cm}$ mass at the dome of the bladder extending anteriorly with trans-serosal involvement and irregular luminal and serosal margins (Fig. 1a). A biopsy of the mass performed via cystoscopy confirmed urachal adenocarcinoma. She then underwent a partial cystectomy to remove the urachus, umbilicus and pelvic lymph nodes. Pathology showed a $6.0 \times 5.3 \mathrm{~cm}$ mucin producing adenocarcinoma, composed of nests of signet ring cells (Fig. 2a, Fig. 2b). Though the margins were negative, the tumour involved the full thickness of the bladder wall, extended to the perivesical fat and serosal surfaces, showed lymphovascular invasion and involved one of four pelvic lymph nodes. There were also several satellite tumour nodules in the perivesical fat. A restaging magnetic resonance imaging (MRI) performed 6 weeks after the partial cystectomy showed a small enhanc- ing mass in the bladder, but this could not be visualized on cystoscopy. A CT scan 6 weeks later confirmed local recurrence (Fig. $1 \mathrm{~b}$ ) and a total cystectomy was planned. However, at the time of cystectomy, there was extensive peritoneal seeding, and as a result a cystectomy was not performed and the patient was referred to medical oncology for further management.

Five months after her initial diagnosis, repeat staging scans showed widespread metastatic disease involving the lung and retroperitoneal lymph nodes (Fig. 1c, Fig. 1d). She began systemic treatment with a standard bladder cancer regimen consisting of gemcitabine and cisplatin. CT scans, performed after 3 cycles (3 months) of treatment, showed progressive retroperitoneal lymphadenopathy, so she was switched to second-line paclitaxel and carboplatin. This regimen was well-tolerated and CT scans done after 3 and 6 cycles showed stable disease, but scans after 9 cycles showed progression in both the lung and the peritoneum. She then received capecitabine and an oral antiangiogenic agent as part of a phase 1 trial. On this regimen, she had initial disease stabilization, but after 6 cycles had significant clinical and radiological progression with the appearance of large adnexal masses, malignant ascites and lesions in the urachal remnant and omentum. She also required bilateral nephrostomy tubes due to ureteric compression from her disease. She came off the trial and started on a single agent, irinotecan, intravenously every 3 weeks. Despite initial symptomatic benefit, further radiologic progression was seen after 3 cycles. She was then given a brief 6 -week course of oral cylophosphamide+prednisone, but the disease progressed. She then had a cycle of capecitabine+oxaliplatin (XELOX), which became available on a compassionate basis, but unfortunately she died of further disease progression.

\section{Discussion}

Urachal cancers are rare and aggressive cancers of the bladder which were originally described by Hue and Jacquin in 


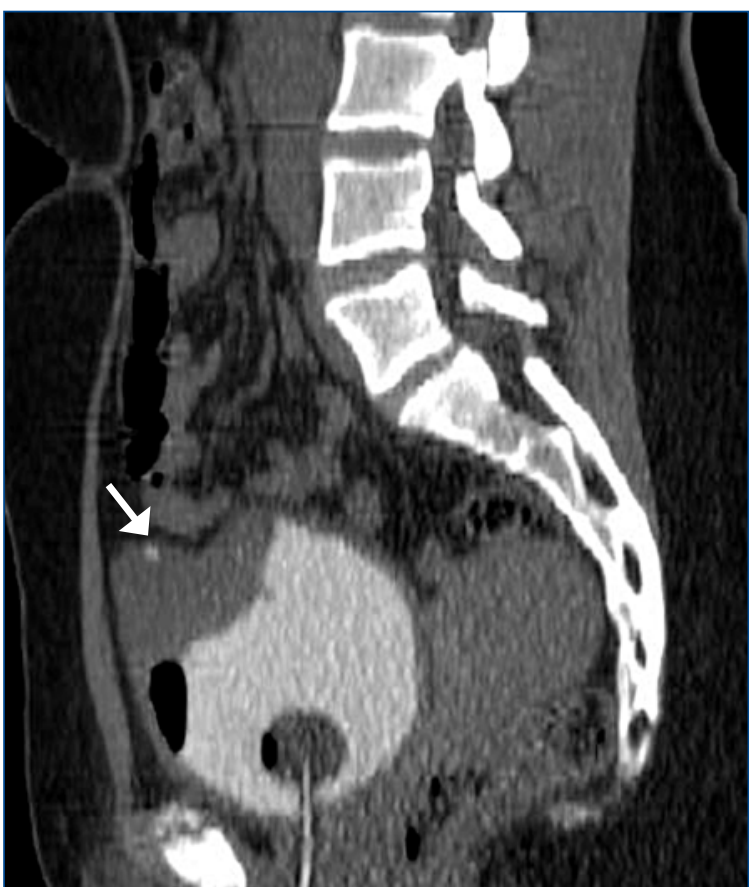

Fig. 1a. Initial diagnosis. Sagittal image from non-contrast computed tomography cystogram shows a soft tissue mass arising from the anterior bladder dome. The mass contains small, coarse calcifications (thick arrow) and is growing outside the expected confines of the bladder wall corresponding anatomically to the urachal remnant.

1863. They account for only $0.5 \%$ of all bladder cancers, and $20 \%$ to $40 \%$ of primary bladder adenocarcinomas. ${ }^{1-3}$ To date, there have been no randomized trials and fewer than 300 cases reported in the literature. As a result, there is a paucity of information on how best to manage these cancers and specifically which chemotherapeutic agents are most effective in an atypical bladder cancer with histological and biological features similar to cancers of the bowel.

Urachal cancers arise from the urachus which is a vestigial embryonic structure located in the space of Retzius, between the transversalis fascia anteriorly and peritoneum posteriorly and between the dome of the bladder and the umbilicus. ${ }^{4,5}$ The urachus is composed of three layers: an outer muscular layer, middle connective tissue layer and an inner layer usually lined with transitional cell epithelium. Most urachal carcinomas are mucin-producing adenocarcinomas which exhibit signet ring cells and an enteric type histology more commonly seen in colon cancers. ${ }^{6}$ The MD Anderson Cancer Center (MDACC) criteria helps in the diagnosis of urachal cancers (Table 1). These criteria include a midline location of the tumour; a sharp demarcation between the tumour and normal surface epithelium; an enteric histology; the absence of urothelial dysplasia, cystitis cystica or cystitis glandularis transitioning to the tumor; and the absence of a primary adenocarcinoma of another origin. ${ }^{7}$

Patients with urachal cancers often present with higher stage disease at diagnosis because the disease arises out-

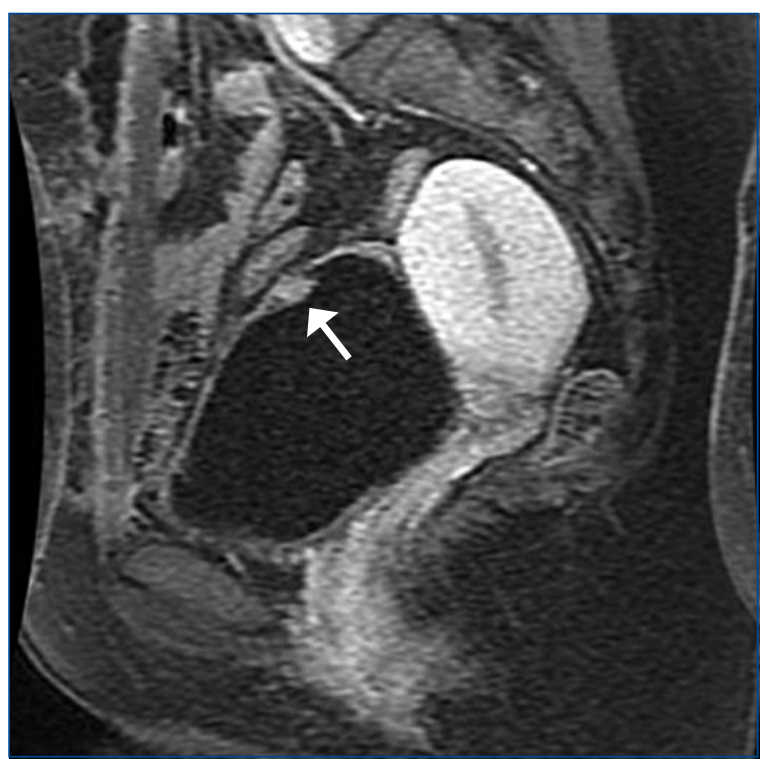

Fig. 1b. Six weeks after partial cystectomy. Sagittal 5-minute delayed post-contrast T1-weighted magnetic resonance imaging sequence with fat saturation shows small enhancing mass at the bladder dome.

side of the bladder where it does not cause any symptoms. Symptoms often occur only after the disease has progressed further and grown into the bladder secondarily. When symptoms occur they commonly include irritative voiding symptoms, discharge of mucous-like material and gross hematuria. Some patients also report umbilical pain and umbilical discharge. ${ }^{7}$ The standard workup includes CT or MRI evaluation of the abdomen and pelvis with a chest $\mathrm{x}$-ray or preferably CT chest to rule out lung metastases. Cystoscopy and an exam under anesthesia are also recommended. As with other enteric malignancies, elevations in tumour markers, including carcinoembryonic antigen (CEA), CA125 and cancer antigen 19-9, have been reported and in some cases a decrease in levels may correlate with response to systemic treatment. ${ }^{8-10}$ If a diagnosis of urachal cancer cannot be a made, a search for an alternate primary oncological diagnosis is recommended.

\section{Table 1. The MD Anderson Cancer Center criteria for the diagnosis of urachal cancer}

\section{Main criteria:}

Location in the bladder dome or elsewhere in the midline of the bladder

Sharp demarcation between tumour and normal surface epithelium

\section{Supportive criteria:}

Enteric type histology

Absence of urothelial dysplasia

Absence of cystitis cystica or cystitis glandularis transitioning to tumour

Absence of primary adenocarcinoma of another origin 


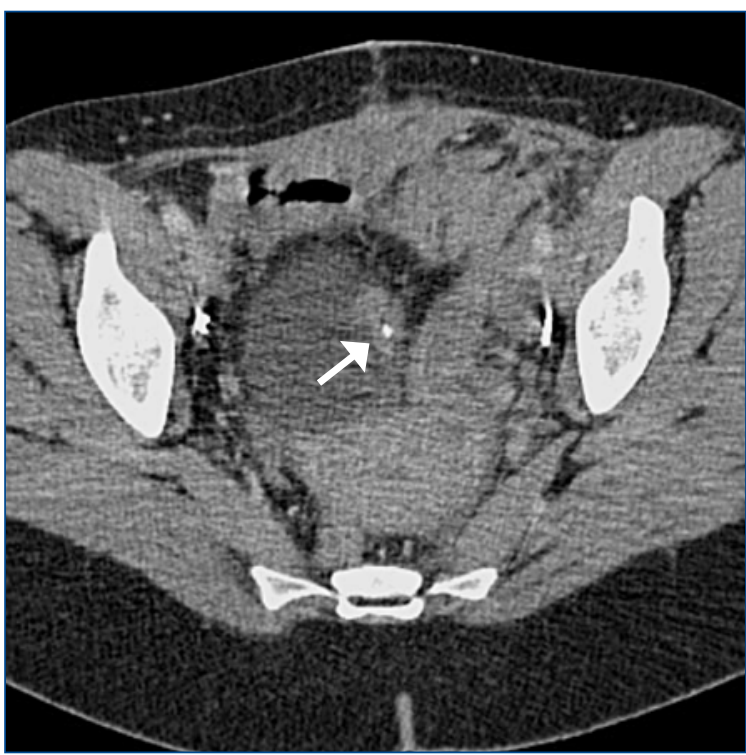

Fig. 1c. Five months after partial cystectomy. Coronal reformatted intravenous contrast-enhanced computed tomography scan reveals a mass at the left anterior bladder dome containing calcifications, compatible with recurrent adenocarcinoma.

An en bloc resection of the urachal ligament and umbilicus is recommended for patients who have surgically resectable disease and either a complete or partial cystectomy ensuring negative margins. This is crucial because urachal tumours can occur anywhere along the urachus, including at the umbilicus $(7 \%)$. If the urachus is transected during surgery, spillage of the tumour containing fluid into the peritoneal cavity can increase the risk of relapse. ${ }^{11,12}$ Currently, an open surgical approach is favoured due to the lack of longterm data on either laparascopic or robotic surgeries. ${ }^{13-15}$

\begin{tabular}{|c|c|c|}
\hline Stage & 1984: Sheldon et al ${ }^{12}$ & 2006: Ashley et al ${ }^{11}$ \\
\hline 1 & $\begin{array}{c}\text { Confined to urachal } \\
\text { mucosa }\end{array}$ & $\begin{array}{c}\text { Confined to urachus and } \\
\text { bladder }\end{array}$ \\
\hline II & $\begin{array}{l}\text { Invasion confined to } \\
\text { urachus }\end{array}$ & $\begin{array}{c}\text { Extension beyond muscularis } \\
\text { or urachus or bladder }\end{array}$ \\
\hline III & - & $\begin{array}{c}\text { Metastatic to regional lymph } \\
\text { nodes }\end{array}$ \\
\hline IIIA & Extension to bladder & - \\
\hline IIIB & $\begin{array}{l}\text { Extension to abdominal } \\
\text { wall }\end{array}$ & - \\
\hline IIIC & $\begin{array}{l}\text { Extension to } \\
\text { peritoneum }\end{array}$ & - \\
\hline IIID & $\begin{array}{l}\text { Extension to other } \\
\text { viscera }\end{array}$ & - \\
\hline IV & - & $\begin{array}{l}\text { Metastatic to nonregional } \\
\text { lymph nodes/distant sites }\end{array}$ \\
\hline IVA & $\begin{array}{l}\text { Metastatic to lymph } \\
\text { nodes }\end{array}$ & - \\
\hline IVB & $\begin{array}{l}\text { Metastatic to distant } \\
\text { sites }\end{array}$ & \\
\hline
\end{tabular}

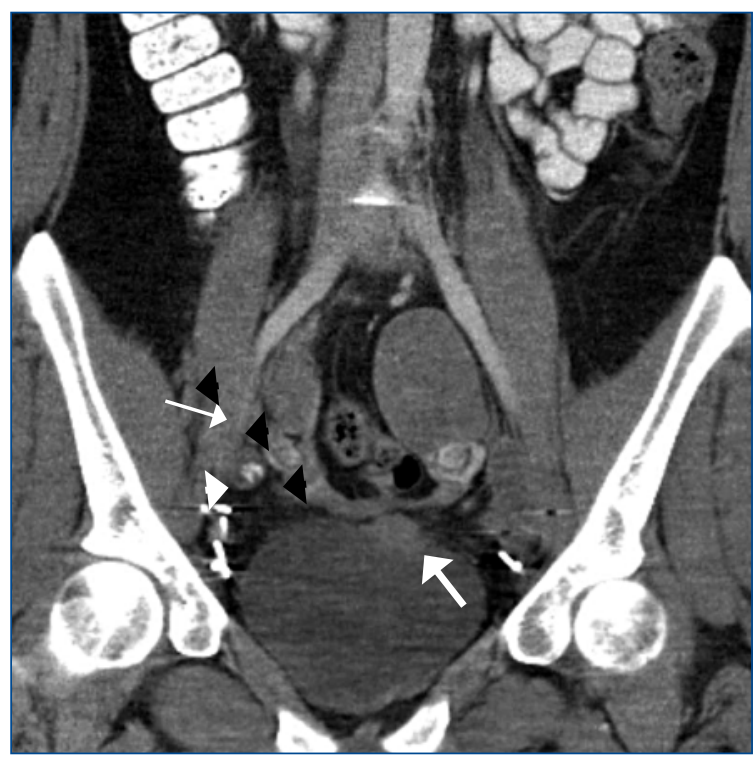

Fig. 1d. Advanced disease. Coronal reformatted intravenous contrast-enhanced computed tomography scan shows left adnexal mass and part of right adnexal mass (thin arrows), metastatic right iliac lymph node with coarse calcification (arrowhead), and part of the left anterior bladder dome mass (thick arrow).

Two systems have been proposed for staging urachal cancers. The first system was proposed by Sheldon and colleagues in 1984. In the Sheldon staging system, early stage urachal cancers are localized to the urachal mucosa, while late stage disease involves the local structures, such as the bladder, abdominal wall or peritoneum, and metastases to regional lymph nodes or distant sites. A more simplified system has been recently proposed by the Mayo clinic, though neither system has been validated (Table 2). ${ }^{11,16}$ The median survival for locally advanced or metastatic disease is between 12 and 24 months and the 5-year cancer specific survival rate for urachal cancers is only $50 \%{ }^{8,11}$

Unlike other cancers, there is currently no standard adjuvant or metastatic chemotherapy regimen for the treatment of urachal cancers. The choice of regimens has been based largely on case reports and single institution experiences (Table 3). Chemotherapy regimens used and available in Canada to treat advanced bladder cancer, including gemcitabine+cisplatin or methotrexate+vinblastine+ doxorubicin+cisplatin (MVAC), have shown a few anecdotal responses in urachal cancers, but have generally yielded disappointing results. ${ }^{7,8,11}$ Given the enteric-type histology of urachal adenocarcinomas, chemotherapy regimens used to treat gastrointestinal malignancies may be more effective. Two case reports have described patients responding to irinotecan-based chemotherapy, a regimen commonly used to treat colorectal cancers. ${ }^{16,17}$ Two case reports using oxaliplatin-based chemotherapy have also shown benefit in urachal cancers. ${ }^{18,19}$ There is one report of a triplet combination of 


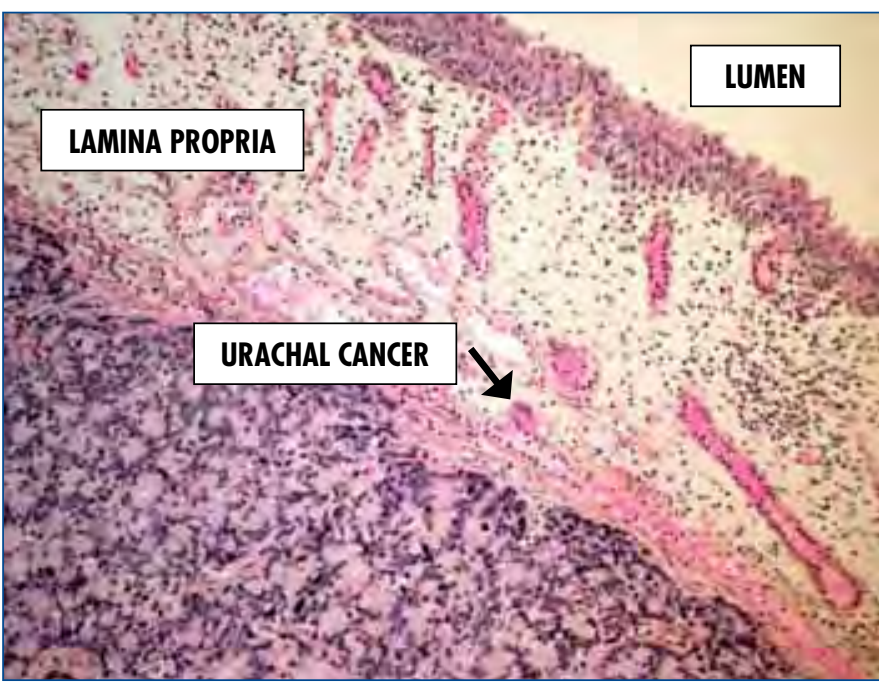

Fig. 2a. Hematoxylin and eosin stain, magnification 2.5×. Urachal adenocarcinoma infiltrating lamina propria of urinary bladder with luminal non-tumorous urothelium.

5-flurouracil+doxorubicin+mitomycin-C showing activity in this disease. ${ }^{20}$ In terms of clinical trials, there was one trial of a triplet combination of ifosfamide+paclitaxel+cisplatin in advanced non-transitional cell carcinoma that has reported some activity in urachal cancers. ${ }^{21}$ There is also a phase II study evaluating gemcitabine+fluorouracil+leucovorin+cispl atin (GemFLP) in metastatic urachal cancer and other adenocarcinomas of the bladder, and preliminary results from this study are encouraging. With the lack of other effective options, this regimen has been adopted as the adjuvant and

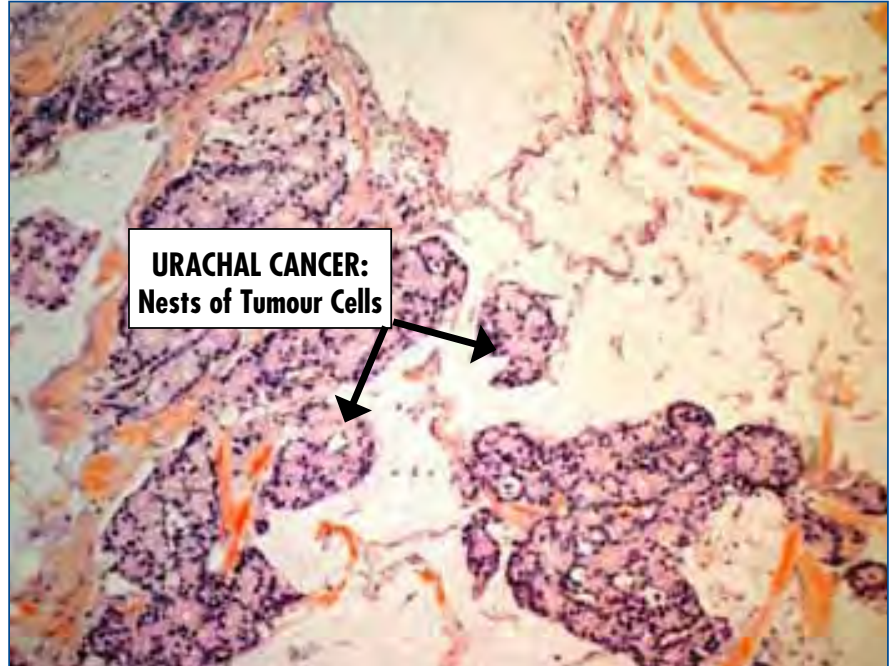

Fig. 2b. Hematoxylin and eosin stain, magnification 2.5×. Urachal mucinous adenocarcinoma, moderately differentiated with groups of tumour cells surrounded by extracellular mucin.

front-line metastatic regimen at the MDACC.

In this case of advanced urachal cancer, taxanes and 5FU-based therapy may have slowed disease progression, similar to reports in larger case series with these agents. The response to taxanes is not surprising since taxanes have demonstrated efficacy in primary adenocarcinomas. ${ }^{21}$ Our patient responded favorably to capecitabine likely based on the enteric histology. She may have had some additional benefit from the antiangiogenic agent, but this would have to be tested in the context of a larger clinical trial. There

Table 3. Chemotherapy regimens tested in urachal cancers

\begin{tabular}{|c|c|c|c|}
\hline Regimen & $\mathbf{N}$ & Setting & Efficacy \\
\hline S- $1+$ cisplatin $\times 5$ courses $^{22}$ & 1 & Metastatic & 1 complete response, disease-free at 30 months \\
\hline S-1+cisplatin ${ }^{23}$ & 1 & Metastatic & 1 response \\
\hline FOLFOX $4^{18}$ & 1 & Perioperative & Minor response to chemotherapy, no recurrence after 1.5 years \\
\hline Irinotecan ${ }^{24}$ & 1 & Metastatic & $\begin{array}{l}\text { Partial response (tumour resistant to cisplatin, 5-FU, doxorubicin, } \\
\text { epirubicin, mitomycin-C) }\end{array}$ \\
\hline $\mathrm{IFL}^{16}$ & 1 & Metastatic & Complete response, recurrence after 6 months \\
\hline Cisplatin+paclitaxel+ifosfamide ${ }^{21}$ & 6 & Advanced & $\begin{array}{c}1 \text { partial response, } 4 \text { with stable disease (duration of response not } \\
\text { reported) }\end{array}$ \\
\hline $\begin{array}{l}\text { 5-FU+doxorubicin+VP16, doxorubicin+ } \\
\text { mitomycin-C+cisplatin }\end{array}$ & 1 & Metastatic & \multirow{2}{*}{$\begin{array}{l}\text { Both survived over } 10 \text { years, chemotherapy was given in the context of } \\
\text { multimodality treatment }\end{array}$} \\
\hline $\begin{array}{l}\text { Doxorubicin+mitomycin-C+ cisplatin, } \\
\text { uracil/ftorafur }{ }^{25}\end{array}$ & 1 & Metastatic & \\
\hline 5-FU+doxorubicin+mitomycin- $\mathrm{C}^{20}$ & 3 & Metastatic & 2 responses \\
\hline Methotrexate+5-FU+epirubicin+cisplatin ${ }^{26,27}$ & 1 & Metastatic & Complete response \\
\hline Ifosphamide+5-FU+VP16+cisplatin ${ }^{27}$ & 4 & Metastatic & 1 complete response, 2 partial response \\
\hline Cisplatin $+5-\mathrm{FU}^{8}$ & 9 & Metastatic & 3/9 partial responses \\
\hline MVAC $^{8}$ & 5 & Metastatic & No response \\
\hline Taxol+methotrexate+cisplatin ${ }^{8}$ & 1 & Metastatic & Response \\
\hline Gem-FLP ${ }^{7}$ ongoing Phase II trial & & Metastatic & 1 complete response, multiple partial responses \\
\hline
\end{tabular}

S-1: oral fluoropyrimidine; FOLFOX4: oxaliplatin $85 \mathrm{mg} / \mathrm{m} 2$ (D1), leucovorin $200 \mathrm{mg} / \mathrm{m} 2$ (D1,2), fluoruracil $400 \mathrm{mg} / \mathrm{m} 2$ (D1, D2), fluorouracil $600 \mathrm{mg} / \mathrm{m} 2$ CIV over 22 hours (D1,2); IFL: irinotecan $125 \mathrm{mg} / \mathrm{m} 2,5 \mathrm{FU} 500 \mathrm{mg} / \mathrm{m} 2$, leucovorin $20 \mathrm{mg} / \mathrm{m} 2$, once weekly for 4 to 6 weeks; MVAC: methotrexate, vinblastine, adriamycin, cisplatin; Gem-FLP: gemcitabine, $5 \mathrm{FU}$, leucovorin, cisplatin. 
was little response to either irinotecan or oxaliplatin, but this could easily have been attributed to the fact that she was already heavily pretreated by the time these agents became available on a compassionate basis. These agents, if available earlier, may have had more benefit. This raises an important issue regarding drug access and availability for patients with rare cancers. Often, there is no phase III clinical trial evidence and biological behaviour may differ from the site of origin necessitating the use of alternate treatment strategies. As we move towards a more personalized approach in oncology, treating according to the histology or other molecular features may improve outcomes in rare cancers such as this one.

\section{Conclusion}

Despite limited evidence, standard chemotherapy for patients with urachal adenocarcinoma should include a taxane or a regimen commonly used to treat gastrointestinal malignancies as opposed to standard transitional cell carcinoma regimens which have not shown much efficacy. The results of the phase II trial of gemcitabine+cisplatin+5FU might further define a treatment standard for this disease. Ultimately, the inclusion of urachal cancer patients in clinical trials will be critical and may also be a mechanism to overcome drug access issues. Studies exploring the use of neoadjuvant, adjuvant or perhaps even intraperitoneal chemotherapy for urachal cancers may lead to better treatment strategies and ultimately better outcomes in this disease.

Competing interests: None declared.

This paper has been peer-reviewed.

\section{References}

1. Munichor $M$, Szvalb S, Cohen H, et al. Mixed adenocarcinoma and neuroendocrine carcinoma arising in the urachus. A case report and review of the literature. Eur Urol 1995;28:345-7.

2. Ghazizadeh M, Yamamoto S, Kurokawa K. Clinical features of urachal carcinoma in Japan: review of 157 patients. Urol Res 1983;5:235-8. http://dx.doi.org/10.1007/BF00272286

3. Wright JL, Porter MP, Li Cl, et al. Differences in survival among patients with urachal and nonurachal adenocarcinomas of the bladder. Cancer 2006;4:721-8. http://dx.doi.org/10.1002/cncr.22059

4. Upadhyay V, Kukkady A. Urachal remnants: an enigma. Eur J Pediatr Surg 2003;13:372-6.

5. Scabini S, Rimini E, Romairone E, et al. Urachal tumour: case report of a poorly understood carcinoma. World I Surg Oncol 2009;7:82. http://dx.doi.org/10.1186/1477-7819-7-82

6. Gopalan A, Sharp DS, Fine SW, et al. Urachal carcinoma: a clinicopathologic analysis of 24 cases with outcome correlation. Am J Surg Pathol 2009;33:659-68. http://dx.doi.org/10.1097/ PAS.0b013e31819aa4ae
7. Siefker-Radtke A. Urachal carcinoma: surgical and chemotherapeutic options. Expert Rev Anticancer Ther 2006;6:1715-21. http://dx.doi.org/10.1586/14737140.6.12.1715

8. Siefker-Radtke A0, Gee J, Shen Y, et al. Multimodality management of urachal carcinoma: the M. D. Anderson Cancer Center experience. J Urol 2003;169:1295-8. http://dx.doi.org/10.1097/01. ju.0000054646.49381.01

9. Kikuno N, Urakami S, Shigeno K, et al. Urachal carcinoma associated with increased carbohydrate antigen 19-9 and carcinoembryonic antigen. J Urol 2001;166:604.

10. Guarnaccia S, Pais V, Grous J, et al. Adenocarcinoma of the urachus associated with elevated levels of CA 125. J Urol 1991;145:140-1.

11. Ashley RA, Inman BA, Sebo TJ, et al. Urachal carcinoma: clinicopathologic features and long-term outcomes of an aggressive malignancy. Cancer 2006;107:712-20. http://dx.doi.org/10.1002/cncr.22060

12. Sheldon CA, Clayman RV, Gonzalez R, et al. Malignant urachal lesions. J Urol 1984;131:1-8.

13. Milhoua PM, Knoll A, Bleustein CB, et al. Laparoscopic partial cystectomy for treatment of adenocarcinoma of the urachus. Urology 2006;67:423.e15-17.

14. Wadhwa P, Kolla SB, Hemal AK. Laparoscopic en bloc partial cystectomy with bilateral pelvic lymphadenectomy for urachal adenocarcinoma. Urology 2006;67:837-43. http://dx.doi.org/10.1016/j. urology.2005.10.048

15. Madeb R, Knopf JK, Nicholson C, et al. The use of robotically assisted surgery for treating urachal anomalies. BJU Int 2006;98:838-42. http://dx.doi.org/10.1111/i.1464-410X.2006.06430.x

16. Mohile SG, Schleicher L, Petrylak DP. Treatment of metastatic urachal carcinoma in an elderly woman. Nat Clin Pract Oncol 2008;5:55-8. http://dx.doi.org/10.1038/ncponc1009

17. Tazi E, Lalya I, Tazi MF, et al. Treatment of metastatic urachal adenocarcinoma in a young woman: a case report. Cases J 2009;2:9145.

18. Kikuchi M, Kamei S, Morirama Y, et al. Case of urachal cancer treated by neoadjuvant chemotherapy with FOLFOX4 (oxaliplatin, 5-FU and leukovolin) [in Japanese]. Hinyokika Kiyo 2008;54:557-9.

19. Tran B, McKendrick J. Metastatic urachal cancer responding to FOLFOX chemotherapy. Can J Urol 2010;17:5120-3.

20. Logothetis $(J$, Samuels ML, Ogden S. Chemotherapy for adenocarcinomas of bladder and urachal origin: 5-fluorouracil, doxorubicin, and mitomycin-C. Urology 1985;26:252-5. http://dx.doi. org/10.1016/0090-4295(85)90121-9

21. Galsky M, lasonas A, Mironov $S$, et al. Prospective trial of ifosfamide, paclitaxel, and cisplatin (ITP) in patient with advanced non-transitional cell (non-TCC) carcinomas of the urothelial tract. Paper presented at: American Society of Clinical Oncology Annual Meeting; June 1-6, 2006; Faiffax, VA.

22. Kojima $Y$, Yamada $Y$, Kamisawa $H$, et al. Complete response of a recurrent advanced urachal carcinoma treated by S-1/cisplatin combination chemotherapy. Int J Urol 2006;13:1123-5. http://dx.doi. org/10.1111/i.1442-2042.2006.01487.x

23. Sekita N, Fujimura $M$, Arai $H$, et al. A case of urachal carcinoma treated with S-1/CDDP combination chemotherapy [in Japanese]. Hinyokika Kiyo 2010;56:447-51.

24. Kume H, Tomita K, Takahashi S, et al. Irinotecan as a new agent for urachal cancer. Urol Int 2006;76:2812. http://dx.doi.org/10.1159/000091635

25. Kawakami S, Kageyama Y, Yonese J, et al. Successful treatment of metastatic adenocarcinoma of the urachus: report of 2 cases with more than 10-year survival. Urology 2001;58:462. http://dx.doi. org/10.1016/50090-4295(01)01259-6

26. Hongoh S, Nomoto T, Kawakami M, et al. Complete response to M-FAP chemotherapy for multiple lung metastases after segmental resection of urachal carcinoma: a case report [in Japanese]. Hinyokika Kiyo 2010;56:107-10.

27. Tatokoro M, Kawakami S, Yonese J, et al. Preliminary report of multimodal treatment with ifosfamide, 5-fluorouracil, etoposide and cisplatin (IFEP chemotherapy) against metastatic adenocarcinoma of the urachus. Int J Urol 2008;9:851-3. http://dx.doi.org/10.1111/j.1442-2042.2008.02113.x

Correspondence: Dr. Srikala S. Sridhar, Medical Oncologist, Princess Margaret Hospital, Assistant Professor, Department of Medicine, University of Toronto, 5222 - 610 University Ave., Toronto, ON M5G 2M9; fax: 416-946-6546; srikala.sridhar@uhn.on.ca 